\title{
Relative importance of energy dependent diffuseness parameter and barrier position in the analysis of fusion excitation function data
}

\author{
Rajesh Kharab a and Manjeet Singh \\ Department of Physics, Kurukshetra University, Kurukshetra, Hr.-136 119, India
}

\begin{abstract}
We have investigated the relative importance of the energy dependence of diffuseness parameter and barrier position in the description of the fusion excitation function data of some heavy ion systems in near barrier energy region. The effects of the energy dependent diffuseness parameter are found to be much more prominent in comparison to those of barrier position.
\end{abstract}

The sub-barrier heavy ion fusion reactions provide a very good insight into nuclear structure and nuclear interaction [1-4]. The interaction potential between heavy ions which consists of Coulomb, centrifugal and nuclear terms plays a very crucial role in the description of fusion dynamics because of the fact that the sum of these terms forms the Coulomb barrier which must be overcome for occurrence of fusion. The Coulomb and centrifugal terms are well understood whereas many ambiguities are associated with the nuclear potential. Since the predictions of fusion excitation functions in sub-barrier energy regions are very sensitive to the shape of nuclear potential, the success of any model for fusion depends strongly on the nuclear potential model employed in the analysis. As a result various potential models ranging from the simple phenomenological to realistic microscopic models have been proposed and used to explain the fusion excitation functions data. Generally, the three parametric Woods-Saxon potential remains the most frequently used potential for the description of compound nuclear reactions. Among the three parameters, depth, range and diffuseness parameter, there exist, large ambiguities in the determination of the accurate value of the diffuseness parameter. It was observed that a wide range of values from $a=0.65 \mathrm{fm}$ to $a=1.5 \mathrm{fm}$ of diffuseness parameter are required to describe various nuclear phenomena [5-12]. As the value of diffuseness parameter increases beyond $a=0.65 \mathrm{fm}$, the potential pocket becomes more and more shallow and disappears for large values and the fusion barrier radius decreases rapidly [6,13-15]. Very recently, an energy dependent parameterization scheme to determine the value of diffuseness parameter was successfully used to explain the fusion excitation functions of various systems [16]. Since, the barrier position changes with the change in the shape of the potential, the energy dependent potential induces energy dependence in the barrier position also.

\footnotetext{
$\overline{{ }^{a} \text { Corresponding author : kharabrajesh@ rediffmail.com }}$
} 
Here we study the relative importance of the energy dependence of the diffuseness parameter and that of the barrier position in the analysis of fusion dynamics. Further, various dynamical effects are properly mocked up by the energy dependent potential, it is sufficient to use the one dimensional barrier penetration model to describe the fusion excitation process. Within the framework of one dimensional barrier penetration model, the Wong's formula for fusion cross-section is very frequently used formula and is written as [17]

$$
\sigma_{F}(E)=\frac{\hbar \omega R_{B}^{2}}{2 E} \ln \left[1+\exp \left(\frac{2 \pi}{\hbar \omega} E-V_{B}\right)\right]
$$

Above $V_{B}, R_{B}$ and $\hbar \omega$ are the barrier height, barrier position and barrier curvature respectively and are important ingredients for calculations. In order to introduce the energy dependence in barrier position, we have replaced $R_{B}$ in the above expression by $R_{E}$ which is written as $[6,14-15]$.

$$
R_{E}=R_{B}-a \ell n\left[1+\exp \frac{2}{V_{B}}\left(E-V_{B}\right)\right]
$$

where ' $a$ ' is the diffuseness parameter and is evaluated by following expression [16].

$$
a(E)=0.85\left[1+\frac{r_{0}}{13.75\left(A_{P}^{-\frac{1}{3}}+A_{T}^{-\frac{1}{3}}\right)\left(1+\exp \left(\frac{\frac{E}{V_{B 0}}-0.96}{0.03}\right)\right)}\right] f m
$$

In the present analysis, we have chosen ${ }_{14}^{28} S i+{ }_{40,92,94} \mathrm{Zr}$ systems and various barrier parameters for these systems, used in the calculations, are listed in Table 1.

Table 1. The values of $V_{B 0}, R_{B}$ and $\hbar \omega$ used in the present calculations for various systems.

\begin{tabular}{|c|rccc|}
\hline System & $\begin{array}{c}V_{B 0} \\
(\mathrm{MeV})\end{array}$ & $\begin{array}{c}R_{B} \\
(\mathrm{fm})\end{array}$ & $\begin{array}{c}\hbar \omega \\
(\mathrm{MeV})\end{array}$ & Reference \\
\hline${ }_{14}^{28} \mathrm{Si}+{ }_{40}^{90} \mathrm{Zr}$ & 74.48 & 9.96 & 3.92 & {$[19]$} \\
\hline${ }_{14}^{28} \mathrm{Si}+{ }_{40}^{92} \mathrm{Zr}$ & 74.20 & 10.00 & 3.97 & {$[18]$} \\
\hline${ }_{14}^{28} \mathrm{Si}+{ }_{40}^{94} \mathrm{Zr}$ & 73.88 & 10.05 & 3.88 & {$[19]$} \\
\hline
\end{tabular}

In Fig.1, we compare calculated sub-barrier fusion excitation functions for ${ }_{14}^{28} \mathrm{Si}+{ }^{90,92,94} \mathrm{Zr}$ systems with the corresponding experimental data. The coupled channel analysis of the fusion of ${ }_{14}^{28} \mathrm{Si}+{ }_{40}^{90} \mathrm{Zr}$ system as indicated in Ref.[20] shows that the effects of the octupole coupling are dominant. In addition, the two-phonon and their mutual couplings are needed to be considered for the good agreement between coupled channel prediction and the fusion excitation function data. However, in this case the transfer channels couplings do not have significant contribution in the enhancement of the fusion excitation function due to fact that $\alpha$ - pickup channel have small positive ground Q-value. 


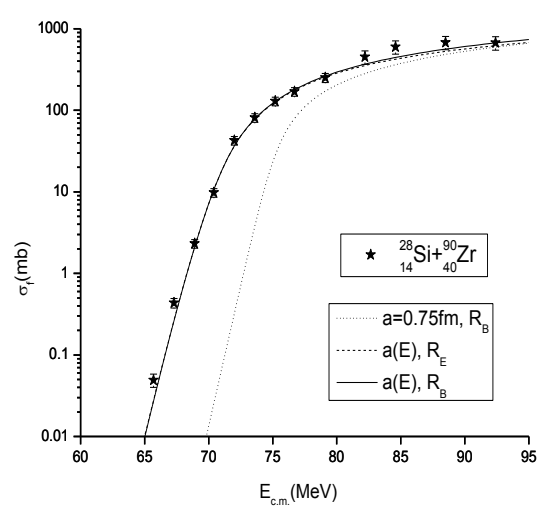

Fig.1a



Fig.1b



Fig.1c

Figure 1. The calculated fusion excitation functions of ${ }_{14}^{28} \mathrm{Si}+{ }_{40,92,94} \mathrm{Zr}$ systems are compared with the corresponding experimental data (*). The experimental data for ${ }_{14}^{28} \mathrm{Si}+{ }_{40}^{90,94} \mathrm{Zr}$ systems are taken from the Ref. [20] while for ${ }_{14}^{28} \mathrm{Si}+{ }_{40}^{92} \mathrm{Zr}$ system are taken from the Ref.[21].

In case of the fusion of ${ }_{14}^{28} \mathrm{Si}+{ }_{40}^{92} \mathrm{Zr}$ system the couplings to double phonon quadrupole as well as octupole vibration states in target ${ }_{40}^{92} \mathrm{Zr}$ and the single phonon $2^{+}$and $3^{-}$vibrational states in projectile and also the inclusion of the two neutron pickup channel having the ground state Q-value equals to $+3.25 \mathrm{MeV}$ is required to explain fusion excitation data within the framework of coupled channel approach [21]. While for ${ }_{14}^{28} \mathrm{Si}+{ }_{40}^{94} \mathrm{Zr}$ system, couplings to the inelastic excitation such as $2^{+}$and $3^{-}$ vibrational states of target and the inclusion of up to four neutron pickup channels are required to explain the energy dependence of the fusion cross-section data [20]. It is found that there is noticeable enhancement in the fusion cross-section in sub-barrier energy regions for ${ }_{14}^{28} \mathrm{Si}+{ }_{40}^{92,94} \mathrm{Zr}$ systems in comparison to ${ }_{14}^{28} \mathrm{Si}+{ }_{40}^{90} \mathrm{Zr}$ system. It may be ascribed to the fact that the additional neutron tries to deform the shape of the nucleus during collision and thus responsible for decrease in the height of the Coulomb barrier resulting in the greater penetration probability. In the Ref.[20], the role of the multinucleon transfer couplings was found to be contributing as we move from the lighter target nuclei to heavier target nuclei. Very recently the coupled channel analysis of the ${ }_{14}^{28} \mathrm{Si}+{ }_{40}^{90,94} \mathrm{Zr}$ systems 
have shown that the effects of one and two protons transfer stripping also play an important role at energies well above the barrier[22]. Here, from the very good agreement between the data and the present prediction in Fig.1, we infer that all these effects are properly imitated by the energy dependent potential and barrier position.

Further, the effects arising due to of the energy dependence of diffuseness parameter are much more pronounced in comparison to that arising because of energy dependent barrier position. It is well known that couplings to various channels lead to a distribution of barriers of varying heights. Similarly the energy dependent nuclear potential results in barriers of varying heights and hence simulates the channel coupling effects and explains the fusion excitation function data reasonably well by using one dimensional barrier penetration model.

To conclude, we have found negligible effects of the energy dependence of the barrier position in comparison to that of the diffuseness parameter in the enhancement of the fusion crosssection data around the barrier over wide range of projectile target combinations.

\section{References}

[1] M. Beckerman, Rep. Prog. Phys. 51, 1047 (1988)

[2] M. Dasgupta, D. J. Hinde, N. Rowley, A. M. Stefanini, Annu. Rev. Nucl. Part. Sci. 48, 401 (1998)

[3] A. B. Balantekin, N. Takigaw, Rev. Mod. Phys. 70, 77 (1998)

[4] L. F. Canto, P. R. S. Gomes, R. Donangelo, M. S. Hussein, Phys. Rep. 424, 1(2006)

[5] K. Hagino, N. Rowley, M. Dasgupta, Phys. Rev. C 67, 054603 (2003)

[6] J. O. Newton et al., Phys. Rev. C 70, 024605 (2004)

[7]K. Hagino et al., Phys. Rev. C 71, 044612 (2005)

[8] I. I. Gontchar et al., Phys. Rev. C 69, 024610 (2004)

[9] J. O. Newton et al., Phys. Lett. B 586, 219 (2004)

[10] J. R. Leigh et al., Phys. Rev. C 52, 3151 (1995)

[11] K. Washiyama et al., Phys. Rev. C 73, 034607 (2006)

[12] A. M. Stefanini et al., Phys. Rev. C 73, 034606 (2006)

[13]J. L. Dethier, Fl. Stancu, Phys. Rev. C 23, 1503 (1981)

[14 ]N. Rowley et al., J. Phys. G 15, L269(1989)

[15] M. Dasgupta et al., Prog. Theor. Phys. Suppl. 154, 209 (2004)

[16] Manjeet Singh, Sukhvinder S. Duhan, Rajesh Kharab, Mod. Phys. Lett.A 26, 2129 (2011)

[17] C. Y. Wong, Phys. Rev. Lett. 31, 766 (1973)

[18] Min. Liu et al., Nucl. Phys. A 768, 80 (2006)

[19] K. Hagino, N. Rowley, A.T. Kruppa, Comput. Phys. Commun. 123, 143 (1999)

[20]S. Kalkal et al., 2010 Phys. Rev. C 81, 044610 (2010)

[21] J. O. Newton et al., Phys. Rev. C 64, 064608 (2001)

[22] S. Kalkal et al., Phys. Rev. C 85, 034606 (2012) 